\title{
Analytical and numerical study of gaseous flow in microchannel with sudden change of section (expansion / contraction)
}

\author{
A Ghodhbane', W Kriaa², A EICafsi' \\ 1. Faculté des Sciences de Tunis (FST), Laboratoire \\ d'Energétique et des Transfert Thermique et Massique \\ (LETTM), Campus universitaire El-Manar, Tunis, Tunisie \\ 2. Ecole Nationale d'Ingénieurs de Tunis (ENIT), \\ Département Génie Industriel, Tunis, Tunisie
}

\begin{abstract}
In this paper, we study an isothermal gas flow (Argon) through a rectangular microchannel with sudden change of section (expansion / contraction). For that, we used two approaches, analytical and numerical, both based on the Navier-Stokes equations, which allows us predicting the pressure driven mass flow rate in the hydrodynamic and slip flow regimes. The analytical approach is elaborated considering the hypothesis of Stokes. The numerical approach is performed using the software Ansys/ Fluent for compressible flow. In the slip regime, the boundary conditions on the wall were added in the Ansys/Fluent software. Both the analytical and numerical mass flow rates show a good agreement in the hydrodynamic and slip flow regimes. The obtained results indicate that the diodicity (the dependence of the mass flow rate from the direction on perfusion) depends on the position of the sections' change in the microchannel, and it is of the order of 2 in our configuration.
\end{abstract}

\section{INTRODUCTION}

In the last decade, the microfluidic domain that outcomes from the MEMS (Micro-ElectroMechanical System) technology, showed a large expansion (Whitesides 2006). The microfluidics englobes different scientific domains, namely, chemistry, biology and aeronautics, etc ... The study goes beyond the simple understanding of the physical phenomena governing the flow to the micrometric scale.

The first investigations were concentrated on the flows through a straight microchannel using different approaches. The analytical approach was made by [Arkilic et al. (1997), Sharipov (1999), Jang et al. (2004), Graur et al. (2006), Dongari et al. (2007), Ghahremani et al. (2008)]. The experimental one by [Arkilic et al. (2001), Colin et al. (2004), Ewart et al. (2006 ; 2007), Graur et al. (2009), Pitakarnnop et al. (2010), P. Perrier et al. (2011), M. Hajd Nacer (2012)]. The main goal of these studies was to determine the mass flow rate, as well as, the accommodation coefficient for a wide range of Knudsen number and for different gases.

Recently, flow through complex geometries has been undertaken by many researches due to their importance in the industrial applications (microturbines, micropumps, etc...). For this reason, it is important to understand the behaviour of the flow through these geometries. In

*Corresponding Author: ghodbaneasma@gmail.com 
this context, a few researches investigated the gas flow through microchannel with longitudinally varying cross section such as Veltzke et al. (2012), Veltzke (2013) and Graur el al. $(2014,2015)$.These authors were interested in the determination of mass flow rate in both flow directions (convergent / divergent) for different gas types and different flow regimes. They showed the presence of diode effect related to the rarefaction level and which has a maximum in the slip flow regime.

Microchannel with sudden change of section (expansion / contraction) were investigated in order to understand the phenomena governing this type of flow. Indeed, A. Agrawal et al. (2005) studied the compressibility and rarefaction effects for a gas flow through a rectangular microchannel with a sudden change of section using the method of Lattice-Boltzmann. They demonstrated the presence of a discontinuity at that part related to the pressure drop in both flow direction (expansion / contraction) caused by the gas compressibility. This discontinuity is accompanied by a jump in velocity that decreases when the Knudsen number increases. A. Agrawal et al. (2005) have deduced that the compressibility and rarefaction of the gas have an opposite effect on the flow. H. Huang et al. (2009), also used the same method but in the case of circular microchannel with sudden change of section. The results shows the presence of vortex whose dimensions decrease when the rarefaction of the gas is more important.

In this study, an analytical and numerical investigation of gaseous flow through a rectangular microchannel with sudden change of section (expansion/ contraction) is undertaken based on the Navier-Stokes equation. These two approaches are developed in order to determine the mass flow rate, the diodicity, etc... and to understand the behavior of the flow in the hydrodynamic and slip regimes.

\section{PROBLEM STATEMENT}

In this work, we study a gas flow through a long microchannel (Figure 1) with a sudden change of section (contraction / expansion). The channel width (w) is assumed constant along the microchannel while the channel height varies suddenly from $h_{1}$ to $h_{2}$ at $X=\frac{3 L}{4}=X_{S}$, with $y\left\{\begin{array}{l}h_{1}, 0 \leq X<X_{s} \\ h_{2}, X_{s} \leq X \leq L\end{array}\right.$ where $\max \left(h_{1}, h_{2}\right)<<w$. The channel is long enough $(L>>w)$ to neglect the end effects. The inlet of the channel is maintained at the pressure $P_{1}$ whereas the pressure in the outlet is $P_{2}$. We also assume the isothermal conditions in the total system.

\section{ANALYTIC APPROCH}

\subsection{Governing equations}

In this section, we develop an analytical model for the microchannel shown in Figure 1.We suppose that the hypothesis of Stokes are verified, so the flow can be modelled as twodimensional. Thus, the Stokes equation is as follows:

$$
\frac{\partial^{2} u}{\partial y^{2}}=\frac{1}{\mu} \frac{d P}{d x}
$$

Where $P=P(x)$ is the local pressure in the microchannel. 


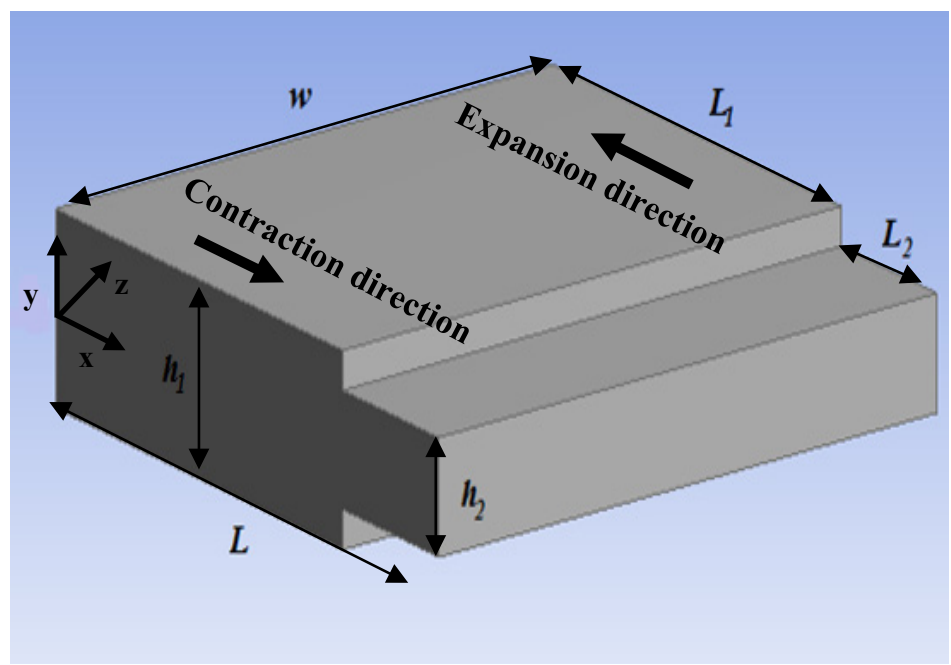

Figure 1: Geometry of microchannel with sudden expansion / contraction.

The gas flow regime in micro size device is characterized by the Knudsen number, which is defined by the ratio between the molecular mean path $(\lambda)$ and the characteristic length $(h)$.

In our work, we define the Knudsen number basing on the mean pressure $P_{m}$.

$$
K n_{m}=\frac{\lambda}{h} \quad \text { where } \quad \lambda=k_{\lambda} \mu \frac{\sqrt{2 R_{s} T}}{P_{m}}
$$

Here $h=h 2_{\min }$ and $P_{m}=\frac{1}{2}\left(P_{1}+P_{2}\right)$

- $k_{\lambda}$ is the coefficient which depends on the molecular interaction model,

- $M$ the mass molar of the fluid,

- $\mu$ is the viscosity coefficient,

- $R_{S}$ is the specific constant gas,

- $T$ is the temperature.

In this study, we consider two flow regimes:

- The hydrodynamic regime: $K n<0.001$,

- The slip regime: $0.001<K n<0.1$.

\subsection{Mass flow rate in hydrodynamic flow regime}

In the hydrodynamic flow regime, the symmetry condition and the non-slip condition at the wall are written as follows:

$$
\left(\frac{\partial u}{\partial y}\right)_{y=0}=0 ; u(x, y)_{y=\left\{\begin{array}{c}
\frac{h_{1}}{2} \\
\frac{h_{2}}{2}
\end{array}\right\}}=0
$$


By integrating twice Equation (1) with boundary conditions expressed by Equation (3), the velocity profile can be expressed by:

$$
u(x, y)=\frac{-1}{2 \mu} \frac{d P}{d x}\left(\left(\frac{h(x)}{2}\right)^{2}-y\right)
$$

The channel height varies as following $h(x)=\left\{\begin{array}{l}h_{1}, 0 \leq X<X_{S} \\ h_{2}, X_{S} \leq X \leq L\end{array}\right\}$

The mass flow rate is defined by:

$$
\dot{M}=2 w \int_{0}^{0.5 h(x)} \rho u(x, y) d y
$$

Taking into account the state equation of the ideal gas $\rho=\frac{P}{R_{S} T}$, by integrating the velocity

$$
\dot{M}=-\frac{2}{3} \frac{w P}{\mu R_{S} T} \frac{d P}{d x}\left(\frac{h(x)}{2}\right)^{3}
$$

profile Equation (4) and using the mass conservation property the mass flow rate is given as:

In the case of the sudden contraction/ expansion, the height $h(x)$ is given as:

$$
\begin{gathered}
h^{C}(x)=h_{1}+H\left(x-X_{S}\right) *\left(h_{2}-h_{1}\right) \\
h^{E}(x)=h_{2}+H\left(x-X_{S}\right) *\left(h_{1}-h_{2}\right)
\end{gathered}
$$

Where $H\left(x-X_{S}\right)$ is the Heaviside function and $X_{S}$ the position of the sudden contraction/expansion in the microchannel. In our case, $X_{s}$ is equal to $3 \mathrm{~L} / 4$.

An appropriate mathematical development gives us the expression of the mass flow rate according to the pressure variation along the microchannel,

$$
\begin{gathered}
\dot{M}^{C}=\frac{w \Delta P P_{m}}{12 \mu R_{s} T} \frac{\left(h_{1} h_{2}\right)^{3}}{\left(X_{S} h_{2}^{3}+\left(L-X_{S}\right) h_{1}^{3}\right)} \\
\dot{M}^{E}=\frac{w \Delta P P_{m}}{12 \mu R_{S} T} \frac{\left(h_{1} h_{2}\right)^{3}}{\left(X_{S} h_{1}^{3}+\left(L-X_{S}\right) h_{2}^{3}\right)}
\end{gathered}
$$

\subsection{Mass flow rate in slip flow regime}

In the slip regime, the boundaries conditions at the wall are written as follows:

$$
\left(\frac{\partial u}{\partial y}\right)_{y=0}=0
$$




$$
u(x, y) \quad y=\left\{\begin{array}{c}
\frac{h_{1}}{2} \\
\frac{h_{2}}{2}
\end{array}\right\}=\frac{\tau_{p}}{k_{\lambda}} K n_{m} h_{2}\left(\frac{\partial u}{\partial y}\right) \quad y=\left\{\begin{array}{c}
\frac{h_{1}}{2} \\
\frac{h_{2}}{2}
\end{array}\right\}
$$

Using the expression of channel height Equation (7) and by integrating the equation (6) of mass flow rate along the microchannel, we obtain in both direction:

$$
\left\{\begin{aligned}
\dot{M}^{C} & =\frac{w \Delta P P_{m}}{\mu R_{s} T} \frac{A B}{\left(L-X_{s}\right) A+X_{s} B} \\
\dot{M}^{E} & =\frac{w \Delta P P_{m}}{\mu R_{S} T} \frac{A B}{\left(L-X_{S}\right) B+X_{S} A}
\end{aligned}\right.
$$

Where;

$$
\begin{gathered}
A=\frac{2}{3}\left(\frac{h_{1}}{2}\right)^{3}-K n_{m} h_{2} A_{1} \frac{h_{1}^{2}}{2} \\
A_{1}=\frac{\tau_{p}}{k_{\lambda}} ; \Delta P=P_{1}-P_{2} \\
B=\frac{2}{3}\left(\frac{h_{2}}{2}\right)^{3}-K n_{m} h_{2} A_{1} \frac{h_{2}^{2}}{2}
\end{gathered}
$$

\subsection{Diodicity}

In order to describe quantitatively the effect of the flow direction on the mass flow rate, we introduce the dimensionless number Diodicity " $D$ ", which presents the ratio of the mass flow rates in contraction and expansion directions to the corresponding $\Delta P$, respectively related to the sudden contraction and expansion.

$$
D=\frac{\frac{\dot{M}_{\text {Contraction }}}{\Delta P_{\text {Contraction }}}}{\frac{\dot{M}_{\text {Expansion }}}{\Delta P_{\text {Expansion }}}}
$$

In our case, the $\Delta P$ are the same in the two directions. In the slip flow regime, the diodicity is a function of Knudsen number.

$$
D=\frac{a+b K n_{m}}{c+d K n_{m}}
$$

$$
\begin{aligned}
& \text { Where } a=\frac{2}{3}\left[X_{S}\left(\frac{h_{1}}{2}\right)^{3}+\left(L-X_{S}\right)\left(\frac{h_{2}}{2}\right)^{3}\right], b=A_{1} * h_{2} *\left(\left(X_{S}-L\right) \frac{h_{2}^{2}}{2}-X_{S} \frac{h_{1}^{2}}{2}\right) \\
& \text { And } c=\frac{2}{3}\left[X_{S}\left(\frac{h_{2}}{2}\right)^{3}+\left(L-X_{S}\right)\left(\frac{h_{1}}{2}\right)^{3}\right], d=A_{1} * h_{2} *\left(\left(X_{S}-L\right) \frac{h_{1}^{2}}{2}-X_{S} \frac{h_{2}^{2}}{2}\right)
\end{aligned}
$$


In the hydrodynamic flow regime, the Knudsen number tends to zero, so the diodicity is a constant equal to:

$$
D=\frac{a}{c}
$$

A comparison between analytical and numerical solution is carried out in the next section.

\section{NUMERICAL APPROACH}

The simulated microchannel (Figure 1) have the characteristic dimensions mentioned in the Table 1. For this dimension we can neglect the influence of the lateral wall along the z-axis (M. Hajd Nacer 2012). Hence, we can adopt a two-dimensional configuration (Figure 2) instead of the three-dimensional geometry (Figure 1). In our case, we placed the sudden change section at the distance equal to $3 \mathrm{~L} / 4$ in the microchannel. The computational grid is generated using Ansys/ Fluent, we used quadratic structured mesh "multizone uniform" (Figure 2). We selected a total number of grids between 500000 and 700000 cells according to the flow direction (expansion / contraction).

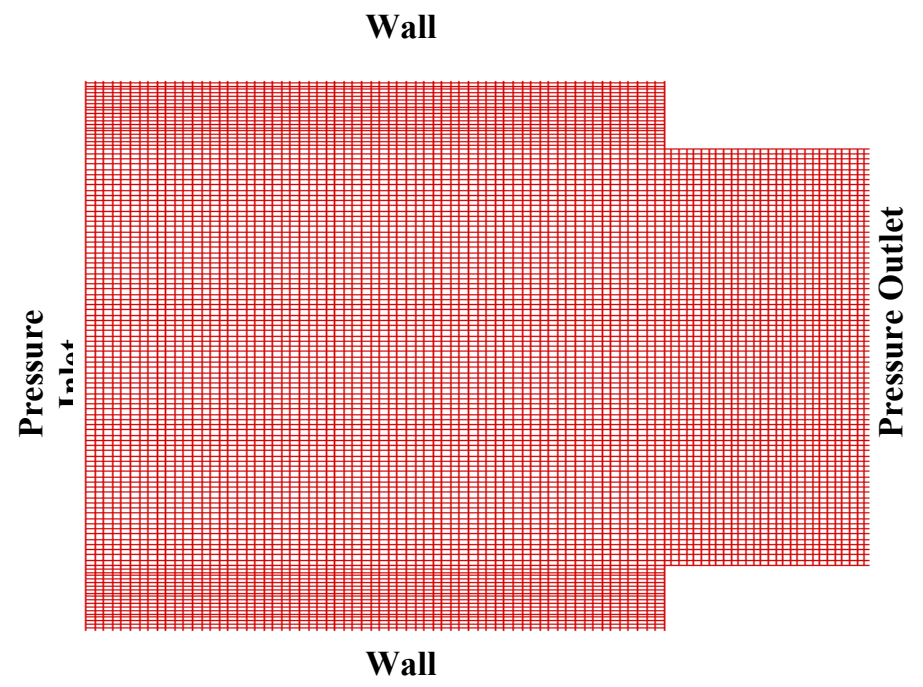

Figure 2: Computational grid for microchannel and the sketch of boundary conditions

Table 1: Microchannel dimensions

\begin{tabular}{ll}
\hline Dimensions & $(\boldsymbol{\mu m})$ \\
\hline L1 & 15000 \\
L2 & 5000 \\
L & 20000 \\
h1 & 100 \\
h2 & 50 \\
W & 2000 \\
\hline
\end{tabular}


The following assumptions were considered:

- The flow is stationary and laminar;

- The configuration is two-dimensional;

- Argon is supposed as compressible gas;

- The force of gravity and the end effects are neglected.

Compressible Navier-Stokes equations and energy equation are used, considering the assumptions above.

Table 2: Thermo-physical proprieties of the gas

\begin{tabular}{cc}
\hline Gas & Argon \\
\hline$k_{\lambda}$ & 0.684 \\
$C_{p}(\mathrm{~J} / \mathrm{kg} . \mathrm{K})$ & 520 \\
$R_{S}(\mathrm{~J} / \mathrm{kg} . \mathrm{K})$ & 208 \\
$\mu_{r e f} 10^{-5}($ Pa.s $)$ & 2.117 \\
$w$ & 0.81 \\
$M(\mathrm{~g} / \mathrm{mol})$ & 40 \\
$T_{r e f}(\mathrm{~K})$ & 273 \\
\hline
\end{tabular}

- The dynamic viscosity of the gas is calculated by $\mu=\mu_{\text {ref }}\left(\frac{T}{T_{\text {ref }}}\right)^{w}$

- $\mathrm{w}$ is the viscosity index depending in the gas nature.

The used boundaries conditions are presented in Figure (2). The tables 3 and 4 explicit the pressure conditions imposed at the inlet and the outlet of the microchannel, respectively for the two regimes, hydrodynamic and slip flow. At the wall, we impose a constant temperature equal to $295.5 \mathrm{~K}$. In the slip flow regime, the slip velocity at the wall is calculated using the Equation (10).

Table 3: Pressure boundary condition for hydrodynamic flow regime

\begin{tabular}{llll}
\hline $\boldsymbol{P}_{\text {in }} \mathbf{( k P a )}$ & $\boldsymbol{P}_{\text {out }}(\mathbf{k P a})$ & $\boldsymbol{\Delta P} \mathbf{( k P a )}$ & $\boldsymbol{K n}_{\text {moy }} \mathbf{1 0}^{-\mathbf{4}}$ \\
\hline 199.982 & 115.152 & 84.830 & 6.867 \\
180.982 & 102.152 & 78.830 & 7.643 \\
165.737 & 90.628 & 75.109 & 8.441 \\
132.600 & 93.300 & 39.300 & 9.580 \\
126.800 & 97.200 & 29.600 & 9.661 \\
125.100 & 97.200 & 27.900 & 9.735 \\
110.250 & 97.200 & 13.050 & 10.000 \\
\hline
\end{tabular}


Table 4: Pressure boundary condition for slip flow regime

\begin{tabular}{llll}
\hline $\boldsymbol{P}_{\text {in }}(\mathbf{k P a})$ & $\boldsymbol{P}_{\text {out }}(\mathbf{k P a})$ & $\boldsymbol{\Delta P}(\mathbf{k P a})$ & $\boldsymbol{K n}_{\text {moy }} \mathbf{1 0}^{\mathbf{2}}$ \\
\hline 45.875 & 4.473 & 41.402 & 0.430 \\
29.850 & 3.504 & 26.346 & 0.648 \\
24.155 & 3.189 & 20.966 & 0.791 \\
18.850 & 3.189 & 15.661 & 0.981 \\
12.155 & 2.100 & 10.055 & 1.518 \\
7.844 & 1.440 & 6.404 & 2.331 \\
\hline
\end{tabular}

This problem was solved with the commercial CFD code Ansys/Fluent based on a finite volume method. The spatial discretization was made with the first order upwind scheme for all the equations of conservation. The pressure-velocity coupling is provided using the SIMPLE algorithm resolution and the convergence criteria was defined when the sum of normalization residues was equal to $10^{-6}$ for all the equations of conservation.

\section{RESULTS AND DISCUSSION}

5.1. Validation of numerical approach in the case of straight microchannel The adopted numerical approach has been validated by comparing the numerical results to the experimental results obtained by M. Hajd Nacer (2012) and analytical results of Graur et al. (2006) for an Argon gas flow with imposed pressure conditions at the inlet and outlet of the microchannel.

The studied microchannel corresponds to the configuration of M. Hajd Nacer (2012). This microchannel have the following dimensions: total length $\mathrm{L}=15006(\mu \mathrm{m})$, constant height $\mathrm{H}$ $=27.91(\mu \mathrm{m})$ and width channel $\mathrm{w}=504.00(\mu \mathrm{m})$. We assume that $h<<w$ to treat the problem as two-dimensional, M. Hajd Nacer (2012) validates this hypothesis. All simulations have been carried out in hydrodynamic and slip regimes.

In the hydrodynamic flow regime, the numerical results of the mass flow rate are compared to the experimental ones obtained by M. Hajd Nacer (2012) and the analytical results of E.B. Arkilic et al. (1997) obtained by the expression below:

$$
\dot{M}=\frac{H^{3} w \Delta P P_{m}}{12 \mu R_{S} T L}
$$

All results are resumed in Table 5. We notice that the analytical, experimental and numerical results of the mass flow rate are in good agreement and the error does not exceed $0.44 \%$. That is considered as a first validation of our numerical model.

Table 5: Pressure boundary condition and mass flow rate for hydrodynamic flow regime $\beta_{\text {Num }}=\left|\left(\dot{M}_{\text {Anal }}-\dot{M}_{\text {Num }}\right) / \dot{M}_{\text {Anal }}\right| ; \beta_{\text {Anal }}=\left|\left(\dot{M}_{\text {Anal }}-\dot{M}_{\text {Exp }}\right) / \dot{M}_{\text {Anal }}\right|$

\begin{tabular}{lll}
\hline $\boldsymbol{P}_{\text {in }} \mathbf{( k P a )}$ & & 125.1 \\
$\boldsymbol{P}_{\text {out }} \mathbf{( k P a )}$ & Numerical (Current study) & 97.2 \\
& Analytical (Arkilic.1997) & 1.354 \\
$\dot{M}\left(\mathbf{1 0}{ }^{-7} \mathbf{k g} / \mathbf{s}\right)$ & Experimental (Nacer.2012) & 1.356 \\
& & 1.350 \\
$\boldsymbol{\beta}_{\text {Num }}(\mathbf{\%})$ & 0.14 \\
$\boldsymbol{\beta}_{\text {anal }} \mathbf{( \% )}$ & & 0.44 \\
\hline
\end{tabular}


As same, for the slip flow regime (table.6), numerical results of the mass flow rate are compared to the analytical on expressed as:

$$
\dot{M}=\frac{H^{3} w \Delta P P_{m}}{12 \mu R_{S} T L}\left(1+6 A_{1} K n_{m}\right)
$$

Where $K n_{m}=k_{\lambda} \mu \frac{\sqrt{2 R_{S} T}}{H P_{m}} ; A_{1}=\frac{\tau_{p}}{k_{\lambda}}$

Table 6: Pressure boundary condition and mass flow rate for slip flow regime.

$$
\frac{\beta=\left|\left(\dot{M}_{\text {num }}-\dot{M}_{\text {Anal }}\right) / \dot{M}_{\text {num }}\right|}{\dot{\boldsymbol{M}}\left(\mathbf{1 0}^{-8} \mathbf{k g} / \mathbf{s}\right)}
$$

\begin{tabular}{lllll}
$\boldsymbol{P}_{\text {in }}(\mathbf{k P a})$ & $\boldsymbol{P}_{\text {out }}(\mathbf{k P a})$ & $\begin{array}{c}\text { Numerical } \\
\text { (Current study) }\end{array}$ & $\begin{array}{c}\text { Analytical } \\
\text { (Arkilic.1997) }\end{array}$ & $\boldsymbol{\beta}(\boldsymbol{\%})$ \\
\hline 1.740 & 1.440 & 0.004381 & 0.004381 & 0 \\
2.610 & 1.440 & 0.1931 & 0.1930 & 0.05 \\
26.440 & 1.440 & 1.710 & 1.699 & 0.64 \\
51.440 & 1.440 & 6.098 & 6.100 & 0.03 \\
\hline
\end{tabular}

We notice a good agreement between analytical and numerical results of the mass flow rate (Figure 3 ) and the error not exceed $0.64 \%$, which allows us to validate our numerical model for the slip flow regime.

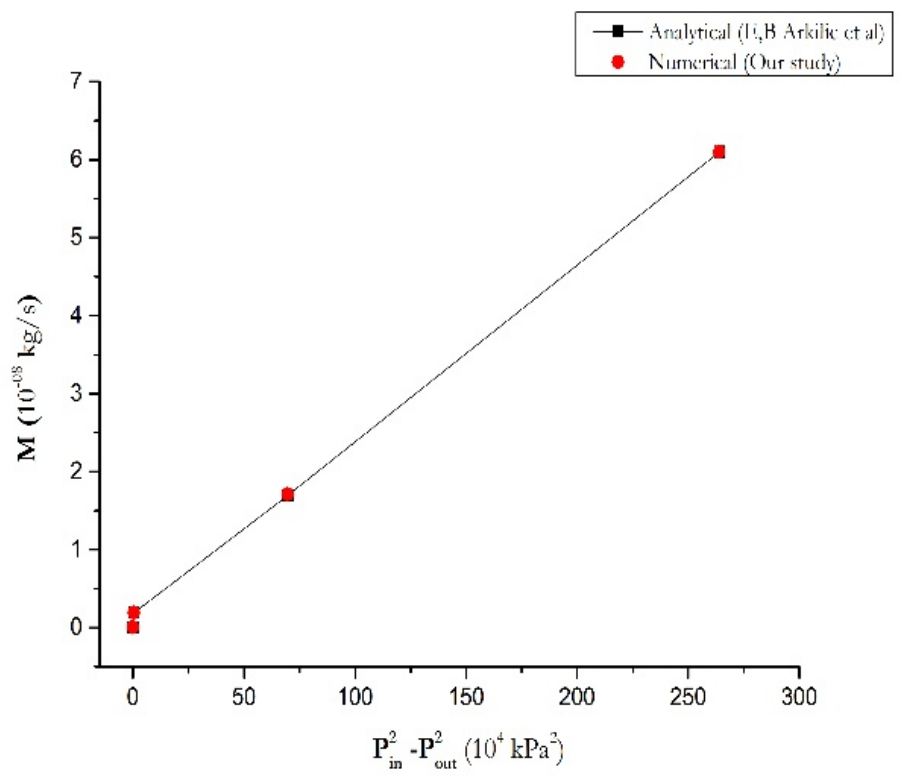

Figure 3: Mass flow rate in slip flow regime for straight microchannel. Solid line with symbol present analytical solution (Arkilic \& al.1997), symbol is our numerical solution 
5.2. Study of microchannel with sudden change section (expansion/ contraction) In this part of work, we are interested in studying Argon flow within a rectangular microchannel sustaining a sudden change of section (expansion / contraction). For that, we opted for an analytical and numerical approach to obtain the mass flow rate, as well as, the other characteristic parameter of the flow for different $\Delta \mathrm{P}$ in the hydrodynamic flow regime (Table 3) and slip flow regime (Table 4). This will help us to identify the effect of the sections' change on the flow behaviour for different means Knudsen numbers.

\subsubsection{Case of sudden change section in the hydrodynamic regime}

Figure 4 illustrate the axial velocity contours and streamlines of the sudden expansion and contraction for two several values of $\Delta P\left(K n_{m} \leq 10^{-3}\right)$.

(a) sudden expansion
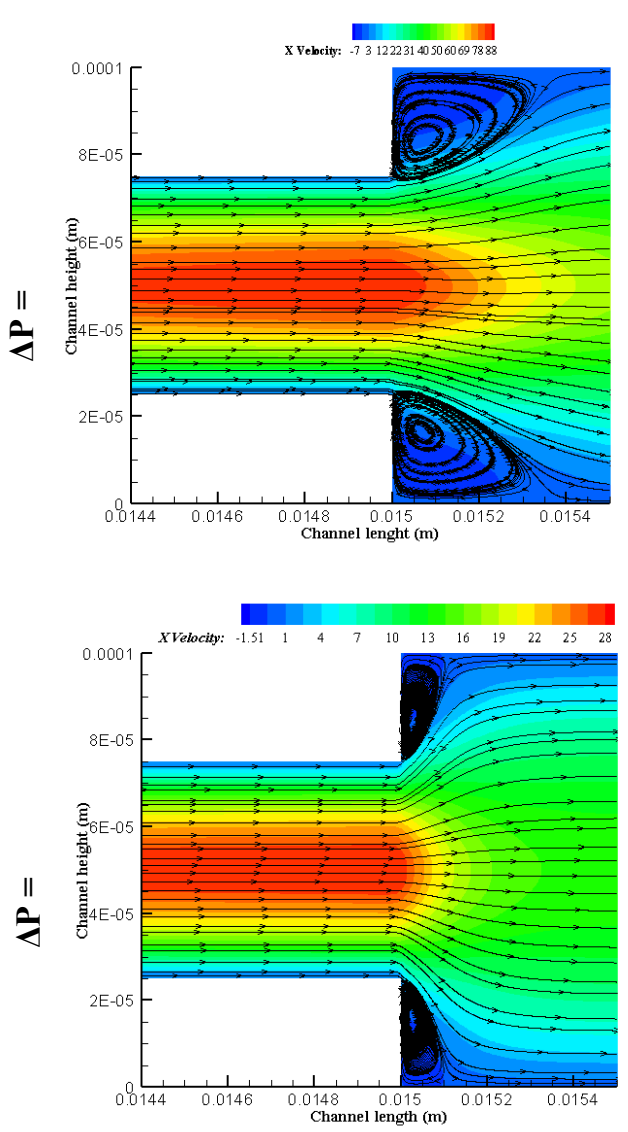

(b) sudden contraction
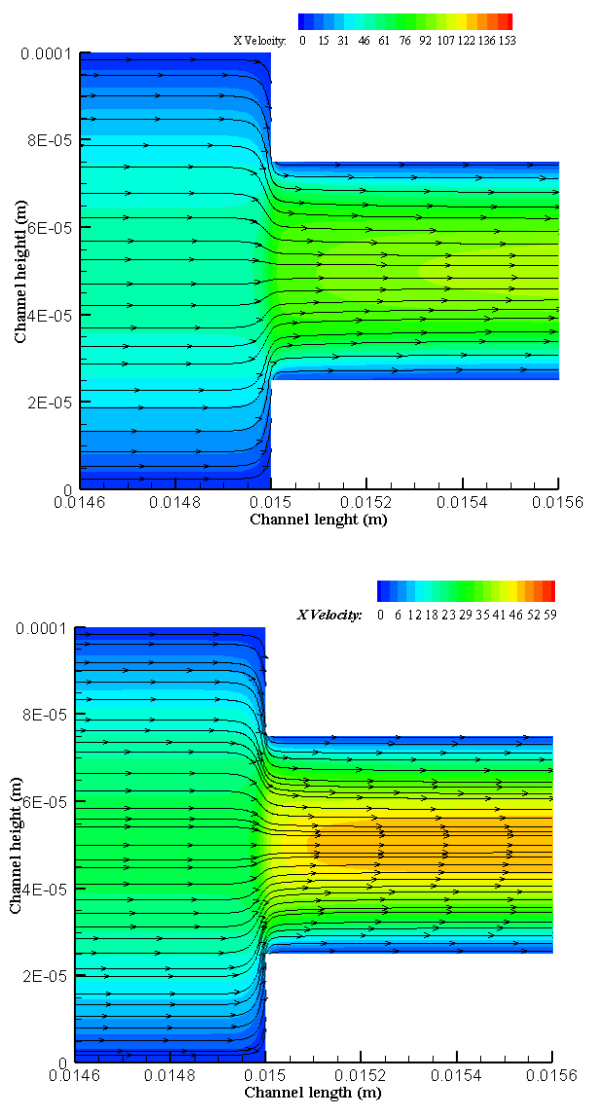

Figure 4: Contours and streamlines of axial velocity for different values of $\Delta P$ (78830 Pa and $27900 \mathrm{~Pa}$ ). (a) Sudden expansion, (b) Sudden contraction 
In the case of the sudden contraction (Figure 4a), we noticed the presence of two symmetric recirculation zones respectively in the upper and lower corner at the change of section. The dimension of the recirculation zones decreases when the difference of the pressure $\Delta \mathrm{P}$ decreases, which means that the flow regime tends to the slip flow regime (number of $K n_{m}$ increase). The vortex disappearances' phenomena for the high mean Knudsen number has been identified by H. Huang et al. (2009) for a circular microchannel under a sudden expansion. Indeed, for the high $K n_{m}$, which correspond to the small pressure gradient $\Delta \mathrm{P}$, the velocity gradient near the wall are less important and that is the origin of the recirculation zone disappearance.

Figure $4 \mathrm{~b}$ shows that, in the case of sudden contraction and for any $\Delta \mathrm{P}$ value, the flow does not contain any vortexes structure. Moreover, the axial velocity is more intense for high $\Delta \mathrm{P}\left(\right.$ low $\left.K n_{m}\right)$ and it is more important in the downstream of the contraction. That is explained by the effect of the sudden change section combined with the effect of compressibility, which causes an acceleration in the velocity flow.

Figure 5 compares the analytical mass flow rate obtained using Equation (8) with the numerical results for different $\Delta P$. We notice a good agreement between the analytical and numerical approach for the mass flow rate until a certain value of $\Delta \mathrm{P}$, from which we observe a big difference between them. This difference may be explained by the high velocity within the microchannel due to the big difference of $\Delta P$ between the inlet and outlet. In this case, the Stokes hypothesis are no longer valid. This is confirmed by the results in Table 7.

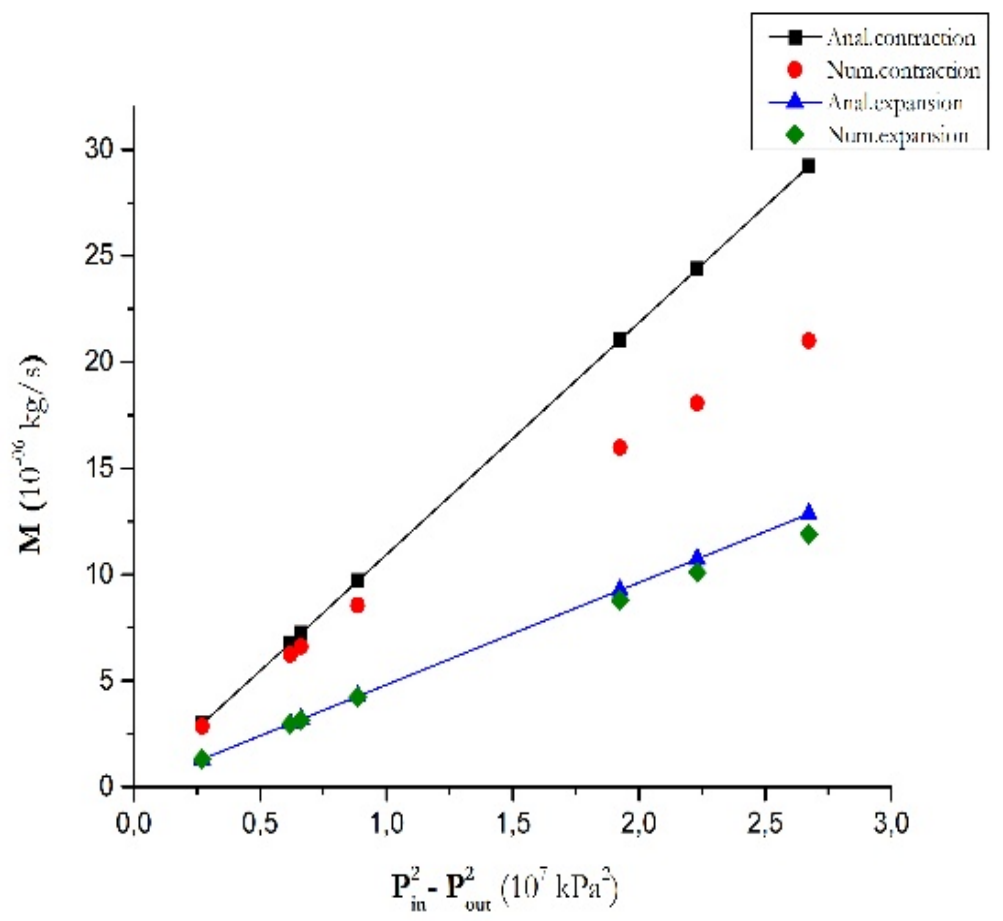

Figure 5: Comparison between analytical mass flow rate obtained by Equation (8) and numerical solution for sudden expansion and contraction in hydrodynamic regime 
Table 7: Comparison between analytical and numerical mass flow rate for hydrodynamic flow regime in direction of sudden expansion.

\begin{tabular}{lllll}
\multicolumn{5}{c}{$\beta=\left|\left(\dot{M}_{\text {Anal }}-\dot{M}_{\text {num }}\right) / \dot{M}_{\text {Anal }}\right|$} \\
\hline $\boldsymbol{P}_{\text {in }} \mathbf{( k P a )}$ & $\boldsymbol{P}_{\text {out }} \mathbf{( k P a )}$ & $\begin{array}{l}\text { Numerical } \\
\text { (current study) }\end{array}$ & $\begin{array}{l}\mathbf{1 0} \text { (10) } \\
\text { Analytical } \\
\text { (current study) }\end{array}$ & $\boldsymbol{\beta ( \% )}$ \\
\hline 199.982 & 115.152 & 11.911 & 12.853 & 7.3 \\
180.982 & 102.152 & 10.104 & 10.731 & 5.8 \\
165.737 & 90.628 & 8.779 & 9.257 & 5.1 \\
132.600 & 93.300 & 4.219 & 4.268 & 1.1 \\
126.800 & 97.200 & 3.163 & 3.187 & 0.7 \\
\hline
\end{tabular}

In the direction of the sudden contraction, Figure 5 shows that the difference between analytical and numerical results of the mass flow rate increases when the pressure gradient becomes more important (high $\Delta P$ ). Table 8 introduces the deviation $\beta$ of the analytical solution compared to the numerical one. In other words, more the difference of pressure was pronounced more the deviation $\beta$ was important and this is due to the compressibility effects (Figure 4). Thus, , we notice that the analytical solution is quite close to the numerical one for low $\Delta P$ when the compressibility effect are limited, that could be explained by the considered hypothesis in the analytical approach which neglected the variation of temperature due to the gas expansion and do not include the energy equation. Also, it is related to the fact that when the Stokes equation is solved (Equation 1) the $U$ velocity (according to $y$ direction) is neglected. However, it is not negligible in the numerical simulation. On the other hand, we noted that the mass flow rate is significantly more important for the sudden contraction compared to the sudden expansion. This is quite reasonable considering that the inlet section of sudden contraction is higher.

Table 8: Comparison between analytical and numerical mass flow rate for hydrodynamic flow regime in direction of sudden contraction.

\begin{tabular}{lllll}
\multicolumn{5}{c}{$\beta=\left(\dot{M}_{\text {Anal }}-\dot{M}_{\text {num }}\right) / \dot{M}_{\text {Anal }}$} \\
\hline $\boldsymbol{P}_{\text {in }} \mathbf{( k P a )}$ & $\boldsymbol{P}_{\text {out }} \mathbf{( k P a )}$ & $\begin{array}{l}\dot{\boldsymbol{M}}\left(\mathbf{1 0}^{-\mathbf{6}} \mathbf{k g} / \mathbf{s}\right) \\
\text { Numerical } \\
\text { (Current study })\end{array}$ & $\begin{array}{l}\text { Analytical } \\
\text { (Current study })\end{array}$ & $\boldsymbol{\beta}$ (\%) \\
\hline 199.982 & 115.152 & 21.014 & 29.211 & 28.0 \\
180.982 & 102.152 & 18.071 & 24.388 & 25.9 \\
165.737 & 90.628 & 15.964 & 21.040 & 24.1 \\
132.600 & 93.300 & 8.548 & 9.700 & 11.8 \\
126.800 & 97.200 & 6.611 & 7.244 & 8.7 \\
125.100 & 97.200 & 6.231 & 6.777 & 8.0 \\
110.250 & 97.200 & 2.843 & 2.958 & 3.8 \\
\hline
\end{tabular}

To quantify the permeability's disparity in the both flow direction (expansion / contraction), we define the diodicity $D$ for the analytical approach given by Equation (14) in the hydrodynamic regime and for the numerical approach, we use Equation (12). All the results for different $\Delta P$ are summarized in Table 9. 
Table 9: Analytical and numerical Diodicity for hydrodynamic flow regime.

\begin{tabular}{llll}
\multicolumn{4}{c}{$\beta=\left|\left(\dot{M}_{\text {Anal }}-\dot{M}_{\text {num }}\right) / \dot{M}_{\text {Anal }}\right|$} \\
\hline $\boldsymbol{P}_{\text {in }}-\boldsymbol{P}_{\text {out }}\left(\mathbf{1 0}^{\mathbf{4}} \mathbf{P a}\right)$ & $\boldsymbol{D}_{\text {num }} \cdot$ & $\boldsymbol{D}_{\text {anal }}$ & $\boldsymbol{\beta} \mathbf{( \% )}$ \\
\hline 8.483 & 1.764 & 2.272 & 22.3 \\
7.883 & 1.788 & 2.272 & 21.3 \\
7.510 & 1.818 & 2.272 & 19.9 \\
3.930 & 2.026 & 2.272 & 10.8 \\
2.960 & 2.090 & 2.272 & 8.0 \\
2.790 & 2.102 & 2.273 & 7.5 \\
1.305 & 2.200 & 2.273 & 3.2 \\
\hline
\end{tabular}

In Figure 6, the diodicity is plotted versus the difference of pressure $\triangle P$. The diodicity obtained by the analytical approach is a constant equal to 2.27 . In contrast, the numerical diodicity evolve linearly and tends to the analytical value for low $\Delta P$. This might be due to the error caused by the Stokes hypothesis, also that the flow is slow, i.e. $U \ll \sqrt{2 R_{S} T}$ and $\operatorname{Re}$ is small.

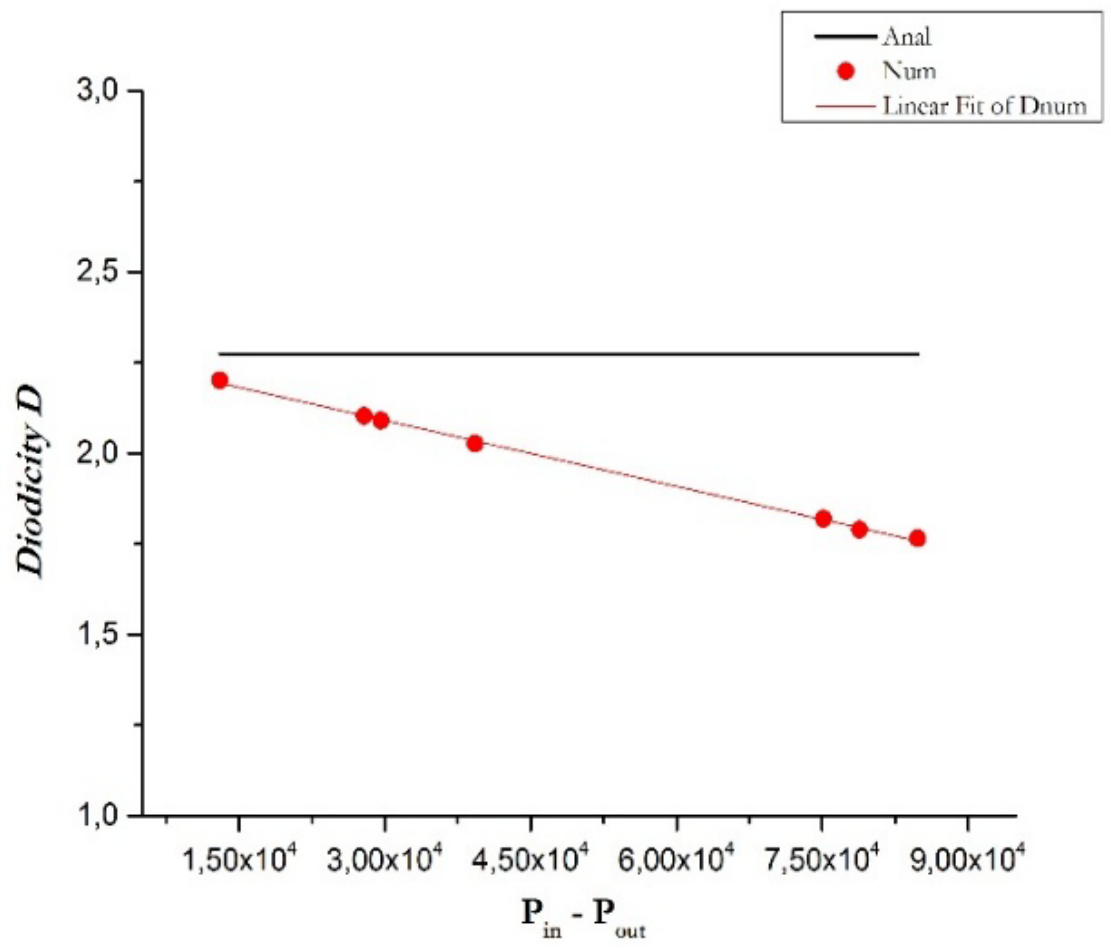

Figure 6: Diodicity as a function of pressure difference in hydrodynamic flow regime. Solid line represents analytical solution (Equation 14), symbol is numerical solution 


\subsubsection{Case of sudden change section in the slip flow regime}

Figure 7 depicts contours and streamlines of the axial velocity for sudden expansion and contraction for several values of average mean Knudsen number $\left(K n_{m}=0.7910^{-2}, 2.3310^{-2}\right)$. In general, the findings given in the hydrodynamic regime are valid in slip flow regime. In fact, we noticed the presence of two recirculation zones in the case of sudden expansion (Figure 7a). These zones' sizes decrease when the average mean Knudsen number increases. In the slip regime, when the average mean Knudsen number increases the compressibility effect decreases whereas the rarefaction effect increases. These two effects act together, hence, the velocity gradient decrease near the wall, which cause the diminution of the vortex dimensions until disappearing.

In the case of sudden contraction (Figure $7 \mathrm{~b}$ ), the flow does not contain a vortexes structures for any Knudsen number and the axial velocity is pronounced when the average mean Knudsen number is lower.

\section{(a) sudden expansion}
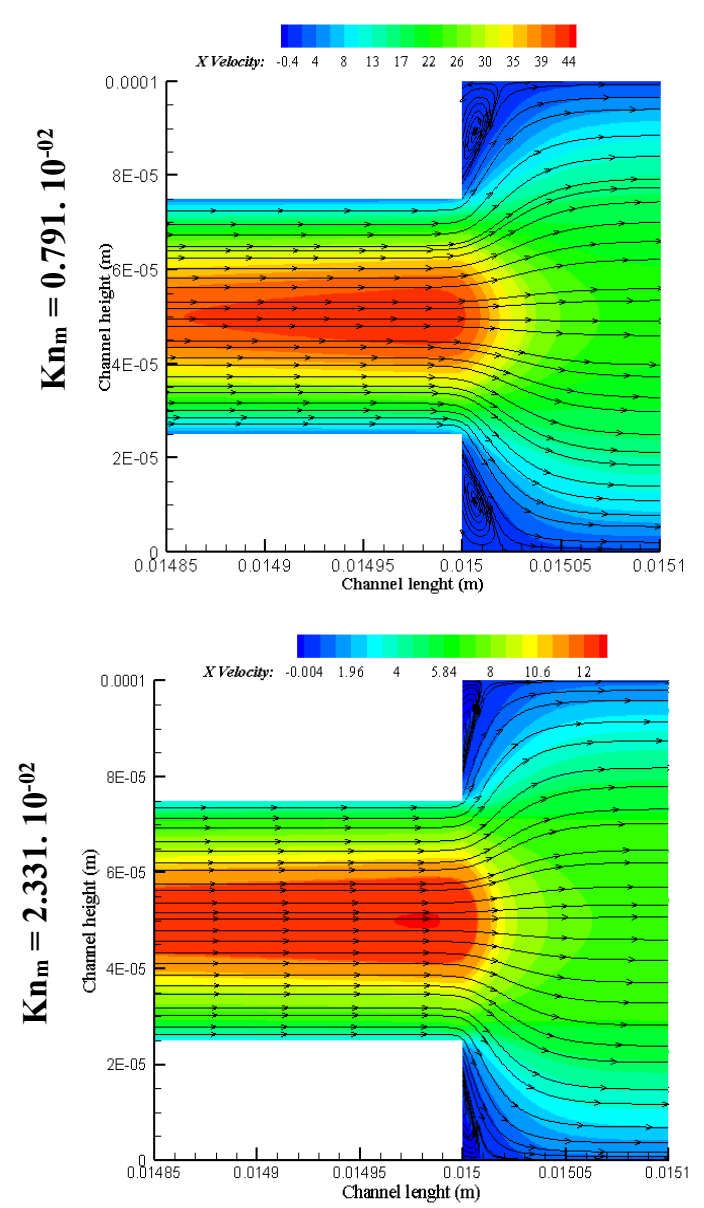

(b) sudden contraction
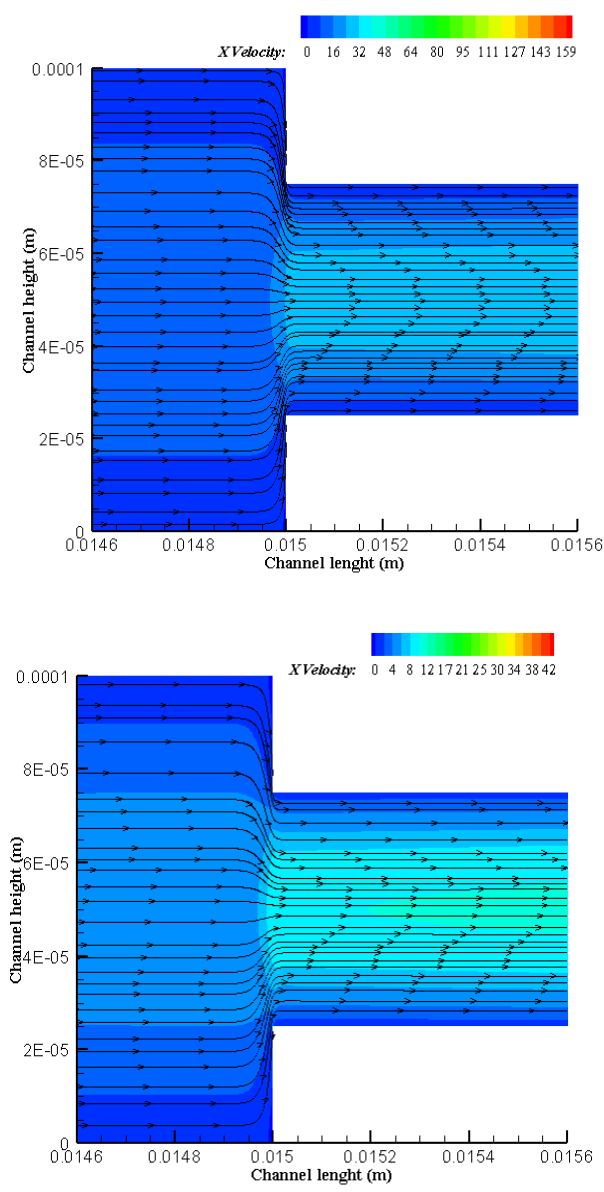

Figure 7: Contours and streamlines of axial velocity for different mean Knudsen number $\left(0.7910^{-02}\right.$ and $\left.2.3310^{-02}\right)$. (a) sudden expansion, (b) sudden contraction 
Expression (11) is used to calculate the analytical mass flow rate in the slip flow respectively for sudden contraction and expansion. The numerical mass flow rate is obtained by our numerical code for different value of $\Delta \mathrm{P}$ and all the results ae summarized in Tables 10 and 11.

Table 10: Comparison between analytical and numerical mass flow rate for slip flow regime in direction of sudden expansion.

\begin{tabular}{lllll}
\multicolumn{5}{c}{$\beta=\left|\left(\dot{M}_{\text {num }}-\dot{M}_{\text {Anal }}\right) / \dot{M}_{\text {num }}\right|$} \\
\hline $\boldsymbol{P}_{\text {in }}(\mathbf{k P a})$ & $\boldsymbol{P}_{\text {out }} \mathbf{( k P a )}$ & $\begin{array}{c}\dot{\boldsymbol{M}}\left(\mathbf{1 0}^{-\mathbf{7}} \mathbf{k g} / \mathbf{s}\right) \\
\text { Numerical } \\
\text { (current study) }\end{array}$ & $\begin{array}{c}\text { Analytical } \\
\text { (current study) }\end{array}$ & $\boldsymbol{\beta}$ (\%) \\
\hline 45.875 & 4.473 & 10.158 & 9.735 & 4.1 \\
29.850 & 3.504 & 4.399 & 4.0568 & 7.8 \\
24.155 & 3.189 & 2.911 & 2.6160 & 10.1 \\
18.850 & 3.189 & 1.782 & 1.5521 & 12.9 \\
12.155 & 2.100 & 0.7728 & 0.6196 & 19.8 \\
7.844 & 1.440 & 0.3407 & 0.24145 & 29.1 \\
\hline
\end{tabular}

Table 11: Comparison between analytical and numerical mass flow rate for slip flow regime in direction of sudden contraction.

\begin{tabular}{lllll}
\multicolumn{5}{c}{$\beta=\left|\left(\dot{M}_{\text {Anal }}-\dot{M}_{\text {num }}\right) / \dot{M}_{\text {Anal }}\right|$} \\
\hline $\boldsymbol{P}_{\text {in }}$ (kPa) & $\boldsymbol{P}_{\text {out }}$ (kPa) & $\begin{array}{c}\dot{\boldsymbol{M}}\left(\mathbf{1 0}^{-\mathbf{7}} \mathbf{~ k g / s )}\right. \\
\text { Numerical } \\
\text { (current study) }\end{array}$ & $\begin{array}{c}\text { Analytical } \\
\text { (current study) }\end{array}$ & $\boldsymbol{\beta}$ (\%) \\
\hline 45.875 & 4.473 & 21.557 & 22.202 & 2.9 \\
29.850 & 3.504 & 9.640 & 9.235 & 4.0 \\
24.155 & 3.189 & 6.460 & 5.972 & 10.0 \\
18.850 & 3.189 & 3.993 & 3.553 & 11.0 \\
12.155 & 2.100 & 1.741 & 1.425 & 18.1 \\
7.844 & 1.440 & 0.768 & 0.559 & 27.2 \\
\hline
\end{tabular}

Figure 8 depicts the evolution of analytical and numerical mass flow rate for sudden expansion and contraction according to the pressure difference $P_{\text {in }}^{2}-P_{\text {out }}^{2}$. We noticed that the mass flow rate in the direction of sudden expansion is lower compared to the sudden contraction, due to the inlet section in the case of sudden contraction that is higher compared to the section of the sudden expansion. Graur et al (2014) observed the similar phenomenon for microchannel with varying cross sections, where the mass flow in nozzle direction is higher compared to the flow in diffusor direction.

This section is devoted to the study of different profiles characterizing the flow in the case of sudden expansion and contraction in the slip flow regime. The pressure distribution at the centreline $(y=0)$ for different values of mean Knudsen number is given in Figure 9. 


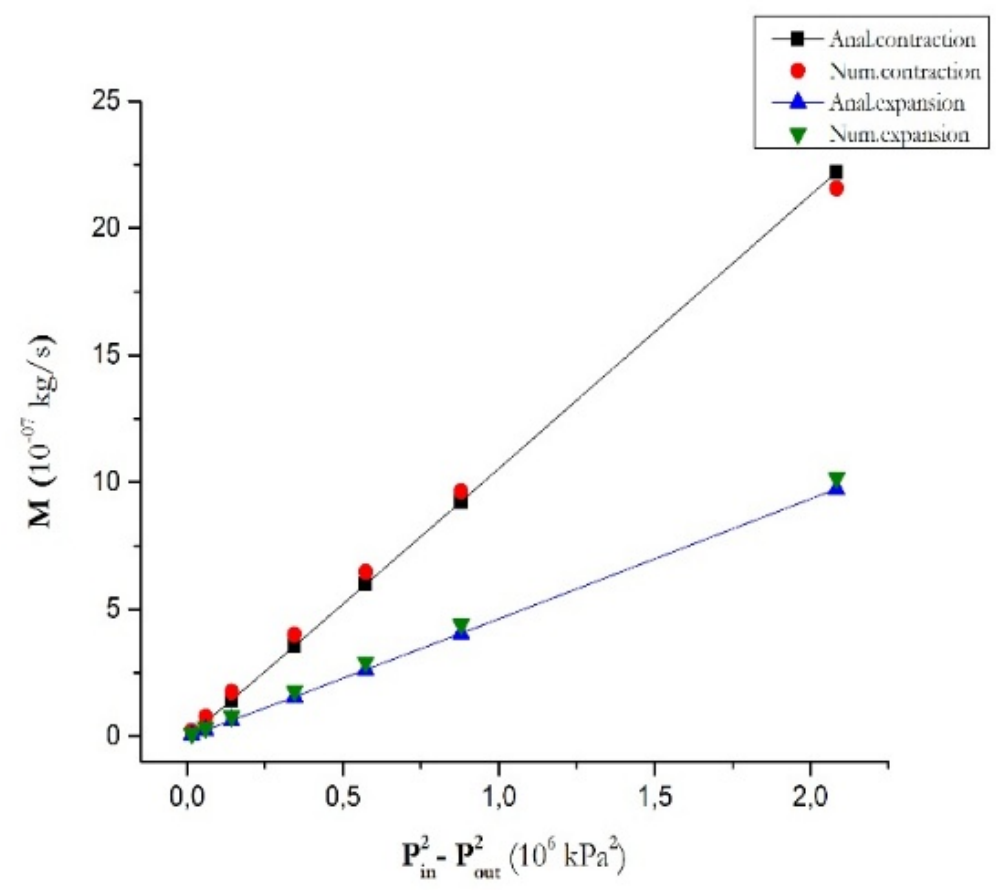

Figure 8: Comparison between analytical mass flow rate obtained by Equation (11) and numerical solution for sudden expansion and contraction in slip regime

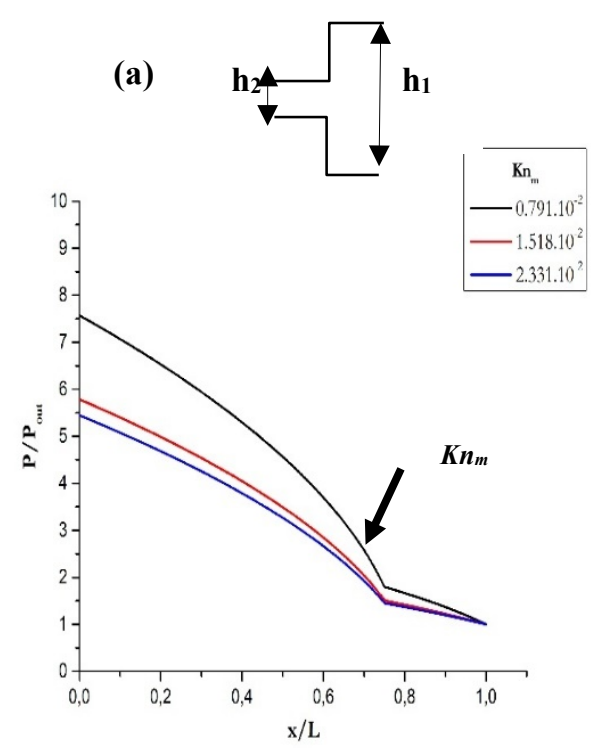

(b)
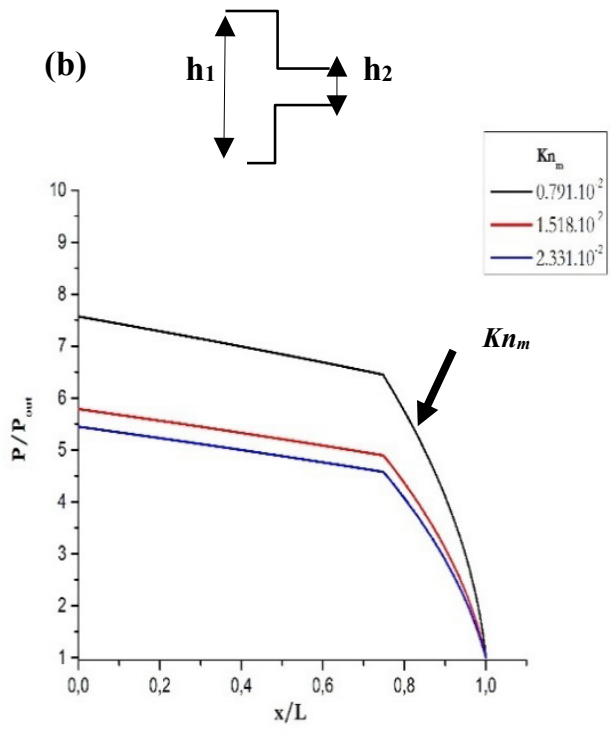

Figure 9: Pressure distribution for different $\left(0.79110^{-02}, 1.51810^{-02}\right.$ and $\left.2.33110^{-02}\right)$.

(a) sudden

mean Knudsen number expansion, (b) sudden contraction 
The pressure evolution shows a discontinuity in slope at the change of section (expansion/ contraction). This discontinuity is due to the effect of compressibility combined with the sudden sections' change (expansion / contraction), this observation agrees with the results of Agrawal et al. (2005). Further, Figure 9 indicates that the pressure distribution is nonlinear in the section of microchannel characterized by low height $h=h_{2}$, against to the rest of the microchannel $\left(h=h_{1}\right)$ the evolution of pressure tends to appear linear. The nonlinearity of pressure is due to the effects of compressibility and rarefaction of gas (Beskok et al 1996), this implies an important acceleration of flow in the section of the microchannel with $\mathrm{h}=\mathrm{h} 2$. As seen in Figure 9, the deviation from a linear behavior decreases with an increase in Knudsen number, therefore, we can deduce that the compressibility and rarefaction have an opposite effect on the evolution of pressure. This agrees with observations of Beskok et al. (1996) and Agrawal et al. (2005).

Figure 10 gives the evolution of the axial velocity $(y=0)$ along the microchannel for sudden expansion and contraction. The difference of pressure imposed between the inlet and outlet of the microchannel combined with the sudden change in cross section creates an acceleration of the flow accompanied by a discontinuity at the change of section (expansion / contraction). The increase in the velocity is due to the effect of compressibility and the change of section. Indeed, Figure 11 shows the decrease of density along the microchannel combined to the reduction in the section, which implies an important acceleration in the velocity (Figure 10). We noted that the jump in velocity decreases as the Knudsen number increase for both direction (sudden contraction and expansion).
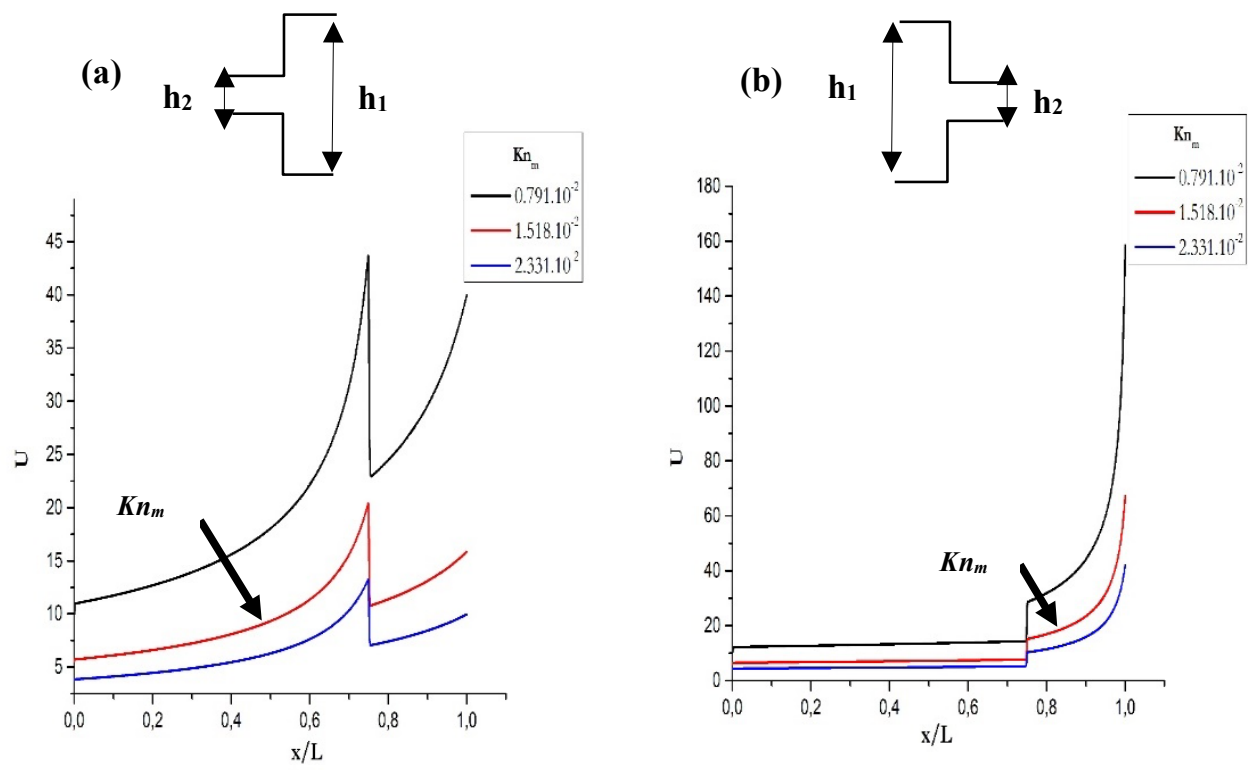

Figure 10: Axial velocity profiles for different mean Knudsen number $\left(0.79110^{-02}, 1.51810^{-02}\right.$ and $\left.2.33110^{-02}\right)$. (a) sudden expansion, (b) sudden contraction 


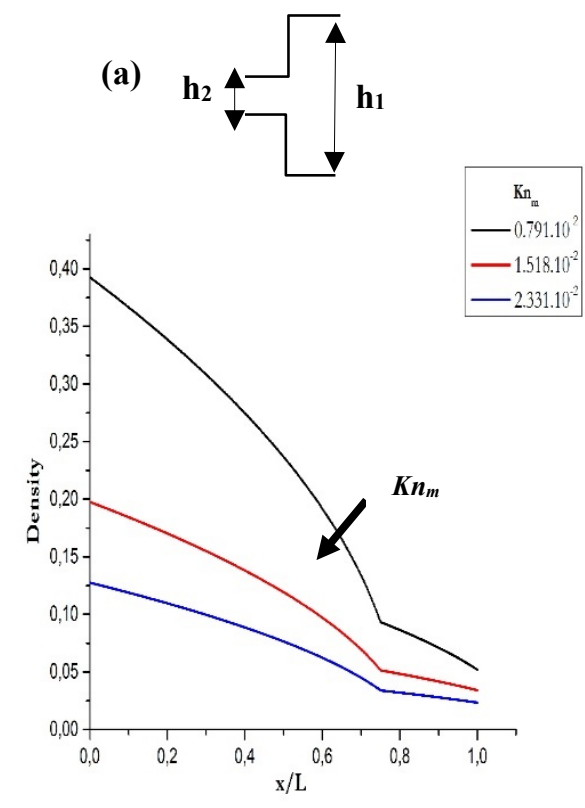

(b)
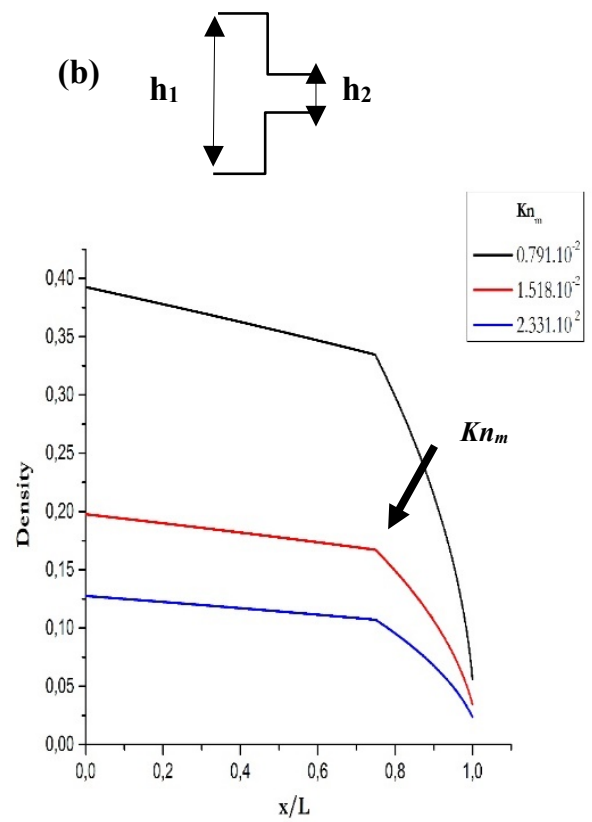

Figure 11: Density profiles for different mean Knudsen number $\left(0.79110^{-02}, 1.51810^{-02}\right.$ and $\left.2.33110^{-02}\right)$. (a) sudden expansion, (b) sudden contraction

In Figure 12, the analytical and numerical diodicity are plotted as a function of mean Knudsen number. It can be seen that the diodicity increases with the gaseous rarefaction in slip flow. We noted that the evolution of analytical and numerical diodicity are in the same order. All results obtained for the diodicity (several values of $K n_{m}$ ), for the analytical and numerical approaches are given in Table 12. We noted that the diodicity is of the order of 2 regardless of the Knudsen number and the error is less than $6.9 \%$. Indeed, we compared our results with the results obtained by Agrawal et al (2005) which have found a diodicity equal to the unity. This can be explained by the effect of the position of the change of section in the microchannel. In fact, Agrawal et al (2005) have positioned their change of section in the middle of microchannel unlike our case where the change of section is placed at $3 \mathrm{~L} / 4$.

Table 12: Analytical and numerical Diodicity for slip flow regime.

\begin{tabular}{llll}
\multicolumn{4}{c}{$\beta=\left|\left(\dot{M}_{\text {Anal }}-\dot{M}_{\text {num }}\right) / \dot{M}_{\text {Anal }}\right|$} \\
\hline $\boldsymbol{K n}_{\text {moy }} \mathbf{1 0}^{-\mathbf{2}}$ & $\boldsymbol{D}_{\text {num }} \boldsymbol{*}$ & $\boldsymbol{D}_{\text {anal }}$. & $\boldsymbol{\beta ( \% )}$ \\
\hline 0.430 & 2.122 & 2.280 & 6.9 \\
0.648 & 2.191 & 2.276 & 3.7 \\
0.791 & 2.219 & 2.282 & 2.7 \\
0.981 & 2.240 & 2.289 & 2.1 \\
1.518 & 2.252 & 2.299 & 1.5 \\
2.331 & 2.254 & 2.315 & 2.6 \\
\hline
\end{tabular}




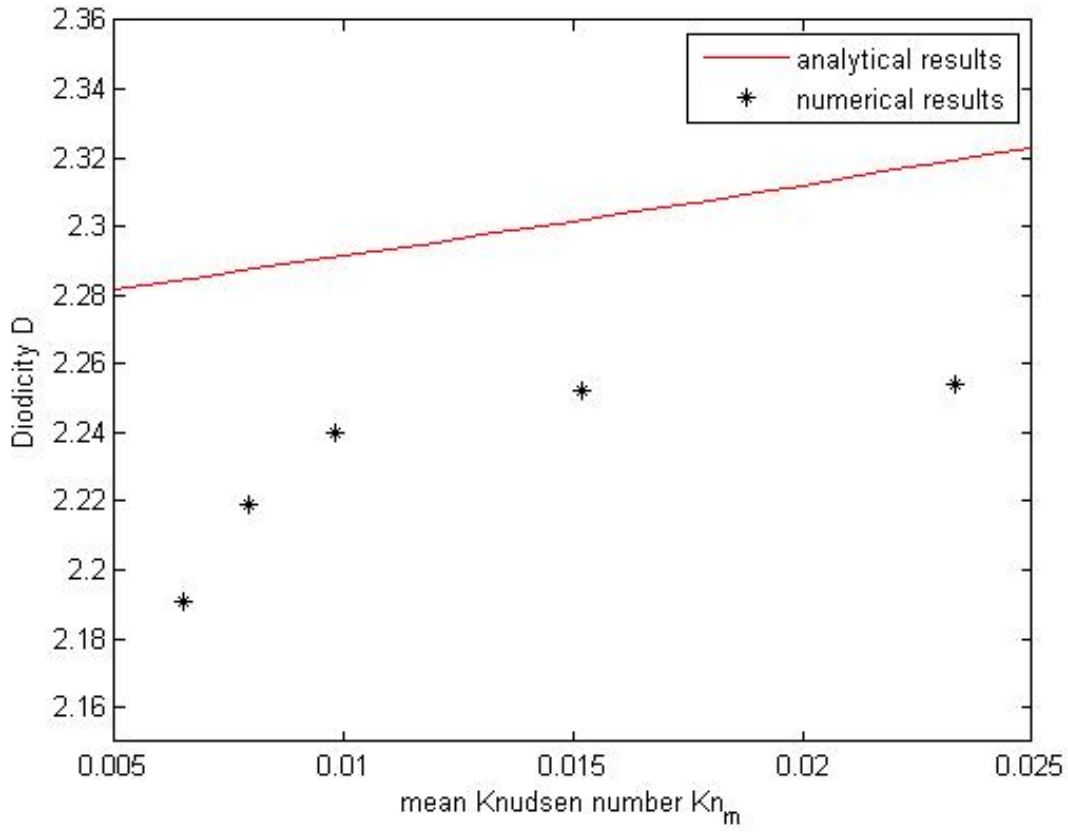

Figure 12: Diodicity evolution versus mean Knudsen number in slip flow regime. Solid line represents analytical solution (Equation 13), symbol is numerical solution

\section{CONCLUSION}

In this work, an analytical and numerical approach has been undertaken for compressible gas (Argon) on a microchannel with sudden change of section (expansion/ contraction). These two approaches based on the solution of Navier-Stokes equations can predict the mass flow rate through these complex geometries. The analytical and numerical results of the mass flow rate are relatively in good agreement. Nevertheless, we note that the mass flow rate in the direction of sudden contraction is higher than the direction of sudden expansion for two flow regimes. In slip flow, the pressure variation shows a discontinuity in slope leading to a jump of the velocity at the sudden change of section (expansion/ contraction). This phenomenon is due to the compressibility effects in the flow. The diodicity is calculated using an analytical and numerical approach for the hydrodynamic and slip flow is found different from unity (of the order to 2). We can explain this effect by the position of the sudden change of section in the microchannel.

\section{REFERENCES}

[1] Agrawal A, Djenidi L, Antonia R., Simulation of gas flow in microchannels with a sudden expansion or contraction. J Fluid Mech 2005 . 530: p. 135-144

[2] Arkilic EB, Schmidt MA, Breuer KS., Gaseous slip flow in long microchannels. J Microelectromech Syst; 1997. 6: p.167-178

[3] Arkilic EB, Breuer KS, Schmidt MA., Mass flow and tangential momentum accommodation in silicon micromachined channels. J Fluid Mech, 2001. 437: p. 29-43

[4] Beskok A, Karniadakis GE, Trimmer W., Rarefaction and compressibility effects in gas microflows. J Fluid Eng, 1996. 118: p. 448-456 
[5] Colin S, Lalonde P, Caen R., Validation of a second- order slip flow model in rectangular microchannels. Heat Transf Eng, 2004. 25: p.23-30

[6] Dongari N, Agrawal A, Agrawal A., Analytical solution of gaseous slip flow in long microchannels. Int J Heat Mass Transf, 2007. 50: p.3411-3421

[7] Dongari N, Sharma A, Durst F., Pressure-driven diffusive gas flows in micro-channels: from the Knudsen to the continuum regimes. Microfluid Nanofluid, 2009. 6: p. 679-692

[8] Ewart T, Perrier P, Graur IA, Méolans JG., Mass flow rate measurements in gas microflows. Exp Fluids, 2006. 41: p.487-498

[9] Ewart T, Perrier P, Graur IA, Méolans JG., Mass flow rate measurements in a Microchannel from hydrodynamic to near free molecular regimes. J Fluid Mech, 2007. 584: p. $1-20$

[10] Ghahremani AR, Mohsenabad SS, Shafii MB., Analytical solution for compressible gas flow inside a two-dimensional poiseuille flow in microchannels with heat flux including the creeping effect. World Academy of Science, Engineering and Technology, 2008. 43: p. $143-147$

[11] Graur IA, Méolans JG, Zeitoun DE., Analytical and numerical description for isothermal gas flows in microchannels. Microfluid Nanofluid, 2006. 2: p.64-77

[12] Graur IA, Perrier P, Ghozlani W, Méolans JG., Measurements of tangential momentum accommodation coefficient for various gases in plane Microchannel. Phys Fluids ,2009. 21:102004

[13] Graur I, Veltzke T, Méolans JG, Ho MT, Thoming J., The gas flow diode effect: theoretical and experimental analysis of moderately rarefied gas flows through a Microchannel with varying cross section. Microfluid Nanofluid, 2014. 18: p. 391-402

[14] Graur I, Ho MT (2015) Rarefied gas flow through a long rectangular channel of variable cross-section. Vaccum 101: 328-332

[15]Huang H, Lu X (2009) Simulation of gas flow in microtubes by Lattice Boltzmann method. Int J Modern Phys C 20: 1145-1153

[16] Jang J, Werely ST (2004) Pressure distributions of gaseous slip flow in straight and uniform rectangular microchannels. Microfluid Nanofluid 1: 41-51

[17] Lockerby DA, Reese JM, Emerson DR, Barber RW., Velocity boundary condition at solid walls in rarefied gas calculations. Phys Review 2004. 70:017303

[18] Nacer MH., Tangential momentum accommodation coefficient in microchannels with different surface materials. PhD thesis, 2012. University of Aix Marseille

[19] Pitakarnnop J, Varoutis S, Valougeorgis D, Geoffroy S, Baldas L, Colin S., A novel experimental setup for gas microflows. Microfluid Nanofluid, 2010. 8: p. 57-72

[20] Perrier P, Graur IA, Ewart T, Méolans JG., Mass flow rate measurements in microtubes: From hydrodynamic to near free molecular regime. Phys Fluids, 2011. 23:042004

[21] Sharipov F., Rarefied gas flow through a long rectangular channel. J Vaccum Sciences and Technology A, 1999. 17: p. 3062-3066

[22] Veltzke T, Baune M, Thöming J., The contribution of diffusion to gas microflow: an experimental study. Phys Fluids, 2012. 24: 082004

[23] Veltzke T., On gaseous microflows under isothermal conditions, 2013. PhD thesis, University of Bremen

[24] Whitesides GM .,The origins and the future of microfluidics, 2006. Nature 442: p. 368373 